\title{
UBVI CCD Photometry of Berkeley 55 Open Cluster
}

\author{
İnci Akkaya Oralhan ${ }^{1, *}$ \\ ${ }^{1}$ Department of Astronomy and Space Sciences, Science Faculty, Erciyes University,TR-38039, Kayseri, Turkey. \\ *Corresponding author. E-mail: iakkaya@erciyes.edu.tr
}

MS received 26 January 2021; accepted 26 January 2021

\begin{abstract}
Fundamental astrophysical parameters have been derived for Be 55 open cluster based on UBVI CCD photometric data, observed with the AZT-22 1.5m telescope at Maidanak Astronomical Observatory in Uzbekistan. The mean reddening is obtained as $\mathrm{E}(\mathrm{B}-\mathrm{V})=1.77 \pm 0.10$ mag from early type members. The zero age main sequence fitting in the $Q_{V \lambda}-Q^{\prime}$ diagrams indicates the distance modulus, $\left(V_{0}-M_{\mathrm{V}}\right)=12.4 \pm 0.20 \mathrm{mag}(\mathrm{d}=3.02 \pm 0.28 \mathrm{kpc})$. This photometric distance is consistent with the distances of Gaia EDR3 $(\mathrm{d}=3.09 \pm 0.16 \mathrm{kpc})$ and period-luminosity relation $(\mathrm{d}=2.78 \pm 0.32 \mathrm{kpc})$ of its Cepheid $S 5$ within the uncertainties. This distance also locates the cluster near the Perseus spiral arm. The Geneva isochrone fittings to the Hertzsprung-Russell diagram and observational colourmagnitude diagrams derive turn-off age, $85 \pm 13 \mathrm{Myr}$, by taking care five red supergiants/bright giants. The possible inconsistences on the locations of the bright giants with the rotating/non-rotating isochrones may be due to both the age spread of stars in young open clusters and the diversity in rotational velocities.
\end{abstract}

Keywords. open clusters and associations: individual Berkeley 55- stars: early-type stars- evolution: late-type supergiants

\section{Introduction}

Be 55 was studied by Negueruela \& Marco (2012) (hereafter N12), Lohr et al. (2018) (hereafter L18), and Alonso-Santiago et al. (2020) (hereafter A20). N12 identified populations of B-type stars, one Ftype and four late-type red supergiants/bright giants (RSGs/RBGs), two Blue Stragglers (BS). Their spectroscopic and photometric analyses indicated an age of $50 \pm 10 \mathrm{Myr}$ and a distance of $\mathrm{d} \approx 4 \mathrm{kpc}$. Its member $S 5$ was identified by L18 as a Type I Cepheid with a pulsation period 5.85 day. Spectral types, effective temperatures and radial velocities of these bright giants have been obtained by A20 from the medium-resolution spectroscopic observations of the $4.2 \mathrm{~m}$ William Herschel Telescope at La Palma. They updated the distance and age of Be 55 as $3.24 \pm 0.22 \mathrm{kpc}$ and $63 \pm 15$ Myr, respectively from PARSEC isochrones (Bressan et al., 2012). Be 55 were also studied by Maciejewski \& Niedzielski (2007), Tadross (2008), Bukowiecki et al. (2011), and Molina Lera et al. (2018), respectively (See Table 7).

As emphasized by A20, Cepheids in young open clusters (OCs) which lie on the blue loop as pulsating variable, have great importance for distance estimation. For their distances, there are useful periodluminosity relations (PLR) in the literature (Benedict et al., 2007; Anderson et al., 2013; Lazovik and Ras- torguev, 2020). Some young OCs with Cepheids as an example are as the following, van den Bergh-Hagen (Marco et al., 2014), Be 51 (Negueruela et al., 2018), NGC 6649 and NGC 6664 (Alonso-Santiago et al. 2020).

Young OCs are generally heavily obscured. For their reddenings, $Q$ technique from their early-type stars is used (Sung et al., 2013) (hereafter S13). For their bright evolved stars, the intrinsic colours of Fernie (1963) and Fitzgerald (1970) for (UBV) and Koornneef (1983) for $\mathrm{JHK}_{s}$ are utilised.

In this paper new Maidanak UBVI CCD Photometry of Be 55 is presented, and analysed by utilising Gaia EDR3 astrometric/photometric data (Brown et al. 7. 2020; Lindegren et al. . 2020). The issues mentioned above have been studied. As emphasized by Lindegren et al. (2020), compared to Gaia DR2 (Brown et al. . 2018; Lindegren et al. , 2018), the average improvement on the uncertainties of parallax/proper motion data of Gaia EDR3 is almost a factor 0.8 for the parallaxes, and 0.5 for the proper motions.

This paper is organized as follows: Section 2 describes the observation and data reduction. The radius, membership selection, the determination of the reddening, and distance modulus/distance are presented in Section 3. Be 55's age plus kinematics/orbital parameters of its bright giants are given in Sections 4-5. A Discussion and Conclusion is presented in Section 6. 
Table 1. For $U B V I$ filters, exposure time, $F W H M$-seeing, the primary/secondary extinction coefficients $\left(k_{1 \lambda}, k_{2 \lambda}\right)$, and the photometric zero-point $\left(\zeta_{\lambda}\right)$.

\begin{tabular}{ccccccc}
\hline Filter & Colour & Exposure time (s) & $F W H M$-seeing $\left({ }^{\prime \prime}\right)$ & $k_{1 \lambda}$ & $k_{2 \lambda}$ & $\zeta_{\lambda}$ \\
\hline U & U-B & 600 & 1.22 & $0.423 \pm 0.022$ & 0.023 & $21.560 \pm 0.011$ \\
B & B-V & 20,600 & 1.99 & $0.324 \pm 0.010$ & 0.026 & $23.261 \pm 0.006$ \\
V & B-V & 10,300 & 0.88 & $0.231 \pm 0.009$ & - & $23.396 \pm 0.001$ \\
I & V-I & 10,120 & 0.74 & $0.139 \pm 0.010$ & - & $23.017 \pm 0.009$ \\
\hline
\end{tabular}

Table 2. Photometric data of Be 55 .

\begin{tabular}{lccccccccc}
\hline$\alpha_{2000}(\mathrm{~h} \mathrm{~m} \mathrm{~s})$ & $\delta_{2000}\left({ }^{\circ \prime \prime}\right)$ & $\mathrm{V}-\mathrm{mag}$ & $(U-B)$ & $(B-V)$ & $(V-I)$ & $\mathrm{eV}$ & $\mathrm{e}(V-I)$ & $\mathrm{e}(B-V)$ & $\mathrm{e}(U-B)$ \\
\hline 211651.27 & 514607.3 & 15.186 & 0.524 & 1.562 & 2.130 & 0.003 & 0.011 & 0.004 & 0.009 \\
21172.30 & 514658.1 & 14.666 & 0.581 & 1.543 & 2.017 & 0.001 & 0.001 & 0.001 & 0.006 \\
21171.72 & 514649.1 & 15.771 & 0.721 & 1.532 & 2.021 & 0.001 & 0.005 & 0.004 & 0.016 \\
$\ldots$ & $\ldots$ & $\ldots$ & $\ldots$ & $\ldots$ & $\ldots$ & $\ldots$ & $\ldots$ & $\ldots$ & $\ldots$ \\
$\ldots$ & $\ldots$ & $\ldots$ & $\ldots$ & $\ldots$ & $\ldots$ & $\ldots$ & $\ldots$ & $\ldots$ & $\ldots$ \\
$\ldots$ & $\ldots$ & $\ldots$ & $\ldots$ & $\ldots$ & $\ldots$ & $\ldots$ & $\ldots$ & $\ldots$ & $\ldots$ \\
\hline
\end{tabular}

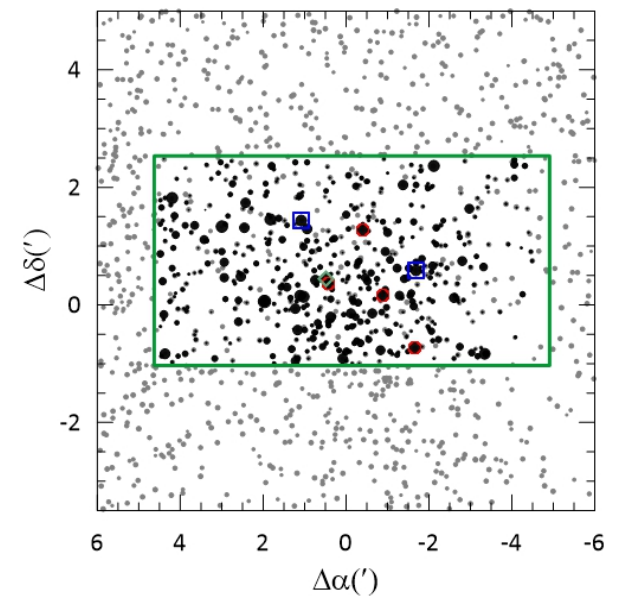

Figure 1. The star chart of Be 55 based on Gaia EDR3 data (grey dots) and Maidanak 2k data (black dots). The position of the stars is relative to its mean equatorial coordinates. Green rectengular shows the field of view of Maidanak detector. Five RSGs/RBGs and two BSs are also indicated (see Fig. 6 for the symbols).

\section{Observation and Data Reduction}

The observation in Johnson-Cousins' $U B V I$ system of Be 55 was carried out with the AZT-22 1.5-m (f/7.74) Ritchey-Chretien telescope at Maidanak Astronomical Observatory (MAO) in Uzbekistan, during high-quality photometric night, on 2005 Agust 9. Images were taken with the SITe $2 k$ CCD detector, which has $2000 \times 800$ pixels, a gain of $1.16 \mathrm{e}^{-} / \mathrm{ADU}$ and a readout noise of 5.3 $\mathrm{e}^{-}$. The combination of the telescope and the detector ensures an unveignetted field of view of $8.85^{\prime} \times 3.55^{\prime}$. Standard magnitude for a given filter $\lambda$ is obtained using the following relation,

$\left.M_{\lambda}=m_{\lambda}-\left[k_{1 \lambda}-k_{2 \lambda} C\right)\right] X+\eta_{\lambda} C+\zeta_{\lambda}$

where $m_{\lambda}, k_{1 \lambda}, k_{2 \lambda}, C$, and $X$ are the observed instrumental magnitude, primary/secondary extinction coefficients, colour index and air mass, respectively. $M_{\lambda}$, $\eta_{\lambda}, \zeta_{\lambda}$ are standard magnitude, transformation coefficient and photometric zero point, respectively. The other details of data reduction can be found in Lim et al. (2009). The SAAO standard stars in Menzies et al. (1991) and Kilkenny et al. (1998) were used to derive atmospheric extinction and transformation coefficients. Pre-processing was performed using the IRAF/ CCDRED package and an aperture of $10^{\prime \prime}$ was used for standard star photometry. Exposure time (s), FWHMseeing ("), extinction coefficients and zero points for $U B V I$ filters are given in Table 1. Here, FWHM-seeing means the seeing, which is estimated from full width at half maximum of the point like stars on the images. The photometric data of 357 stars are listed in Table 2 for a sample data.

Be 55'star chart is displayed in Fig. 1. Its mean central equatorial (J2000) and the Galactic coordinates are as the following, $\mathrm{RA}=21^{h} 16^{m} 58^{s} .01, \mathrm{Dec}=+51^{\circ}$ $45^{\prime} 32^{\prime \prime} .04 ; \ell=93^{\circ} .027, b=1^{\circ} .798$, respectively.

The mean photometric errors of $V$ mag, the colour indices $(V-I),(B-V)$ and $(U-B)$ against $V$-mag for Be 55 are presented in Table 3. The photometry for Be 55 is compared to the $U B V C C D$ photometry of N12. For 26 common stars, the differences of $\Delta V$ and $\Delta(B-V)$ against $(B-V)$, and the difference of $\Delta(U-B)$ against $(U-B)$ are displayed in Fig. 2. When the large deviant data of the evolved stars are excluded from the 
Table 3. The mean photometric errors of $<V>$ mag, $<(V-I)\rangle,\langle(B-V)>$ and $\langle(U-B)>$ against $V$-mag.

\begin{tabular}{ccccc}
\hline$V$ & $\left\langle\sigma_{V}\right\rangle$ & $\left\langle\sigma_{V-I}\right\rangle$ & $\left\langle\sigma_{B-V}\right\rangle$ & $\left\langle\sigma_{U-B}\right\rangle$ \\
\hline $13-14$ & 0.003 & 0.013 & 0.008 & 0.005 \\
$14-15$ & 0.005 & 0.020 & 0.007 & 0.016 \\
$15-16$ & 0.008 & 0.007 & 0.007 & 0.017 \\
$16-17$ & 0.005 & 0.007 & 0.009 & 0.032 \\
$17-18$ & 0.006 & 0.009 & 0.010 & 0.065 \\
$18-19$ & 0.012 & 0.016 & 0.023 & 0.129 \\
$19-20$ & 0.016 & 0.021 & 0.053 & - \\
$20-21$ & 0.050 & 0.052 & 0.123 & - \\
$21-22$ & 0.090 & 0.095 & 0.156 & - \\
$22-23$ & 0.150 & 0.157 & - & - \\
\hline
\end{tabular}

calculation, the mean differences together dispersions of $V$ and $(B-V)$ are $\Delta V=+0.036 \pm 0.027 \mathrm{mag}$ and $\Delta(B-V)=+0.003 \pm 0.012$, respectively. The difference, $\Delta(U-B)$ is $+0.082 \pm 0.064$, which is systematically bluer than N12. For the interval of $14<V<18 \mathrm{mag}$, the $V$ magnitudes of N12 seem to be slightly fainter. For $14<V<18 \mathrm{mag},(B-V)$ values of this paper are in good consistent with $\mathrm{N} 12$, except for bright giants. In panel (c) there appears a discrepancy between -0.4 to -1.0 in $(U-B)$ of four RBGs/RSGs between N12 and Maidanak observations. For Cepheid S5 (red diamond), the discrepancy is about $0.50 \mathrm{mag}$ in $\Delta V$ and $0.2 \mathrm{mag}$ in $\Delta(B-V)$ from panels (a) and (b). The reason for large colour difference for four late-type supergiants with very red colours between $(U-B)$ of present photometry and N12 is due to the so-called red leak in the used $U$ filter. Therefore, their $U$-magnitudes of present photometry are brighter than N12. However, there is no any red leak effect in the used $U$ filter, as is seen from table 2 of the transformation coefficients of Lim et al. (2009).

\section{Fundamental Parameters of Be 55}

\subsection{Radius of Be 55}

For the size of Be 55, its stellar radial density profile (RDP) (Fig. 3) is constructed from the Gaia EDR3 photometric/astrometric data for the field and cluster members within $15^{\prime} .0$, down to $G=20$ mag. Its RDP has been constructed by counting stars in concentric rings of increasing width with distance to its centre. The number and width of rings were optimised so that the resulting RDP had adequate spatial resolution with moderate $1 \sigma$ Poisson errors (Bonatto and Bica , 2007). The solid curve (Fig. 3) denotes the fitted King's profile (King , 1966). The two-parameter function, $\sigma(R)=\sigma_{b g}+\sigma_{0} /\left(1+\left(R / R_{c}\right)^{2}\right)$ is adopted. Here, $\sigma_{b g}$ is the residual background density, $\sigma_{0}$ the central density of stars, and $\mathrm{R}_{\text {core }}$ the core radius. The
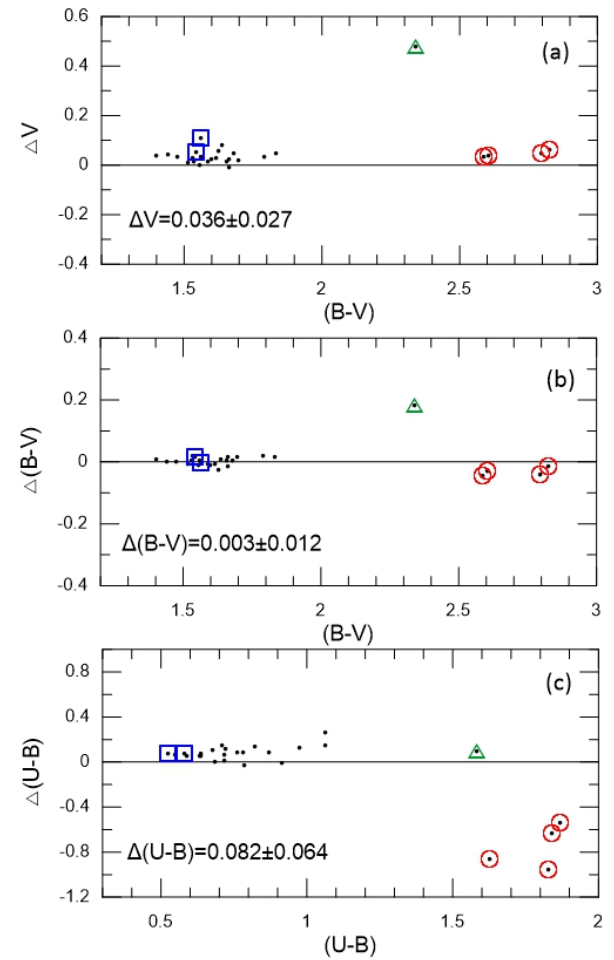

Figure 2. The differences of $V \operatorname{mag},(B-V)$ and $(U-B)$ as a function of $(B-V)$ and $(U-B) . \Delta$ means this paper N12. Five RSGs/RBGs are plotted for just presentation (for the symbols, see Fig. 6).

horizontal gray bar shows the stellar background level measured in the comparison field. From Fig. 3, the core and cluster radii have been determined as $\left(R_{\text {core }}, R_{R D P}\right)$ $=\left(0^{\prime} .45,2^{\prime} .5\right)$. The cluster radius is compatible with $2^{\prime} .5$ of Molina Lera et al. (2018) and $2^{\prime} .2$ of Sampedro et al. (2017). $R_{\text {core }}$ value of this paper is less than $1^{\prime} .32$ of Bukowiecki et al. (2011), $0^{\prime} .90$ of Kharchenko et al. (2013), and $1^{\prime} .28$ of A20, respectively.

\subsection{Membership Selection}

As is seen in the papers of Akkaya Oralhan et al. (2019) and Akkaya Oralhan et al. (2020), UBVI photometric data of Be 55 have been combined with Gaia EDR3 proper motion and parallax data (Brown et al. 2020; Lindegren et al. , 2020) to classify the likely cluster members. On the $\left(\mu_{\alpha}, \mu_{\delta}\right)$ plane (Fig. 4), 305 stars (filled dots) within $R_{R D P}=2^{\prime} .5$ are displayed. The grey dots represent the field background/foreground stars with a region centered around $15 \mathrm{arcmin}$. The fitted

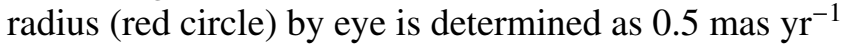
by utilising the histograms (shown with red lines) attached to the $\mu_{\alpha}$ versus $\mu_{\delta}$ plot. 109 probable members in the red circle are used to calculate from both the median values $\left(<\mu_{\alpha}>\right.$ and $\left\langle\mu_{\delta}>\right)$ and the quantity $\left.\mu_{\mathrm{R}}=\sqrt{\left(\mu_{\alpha}-<\mu_{\alpha}>\right)^{2}+\left(\mu_{\delta}-<\mu_{\delta}>\right)^{2}}\right)$. Parallax 


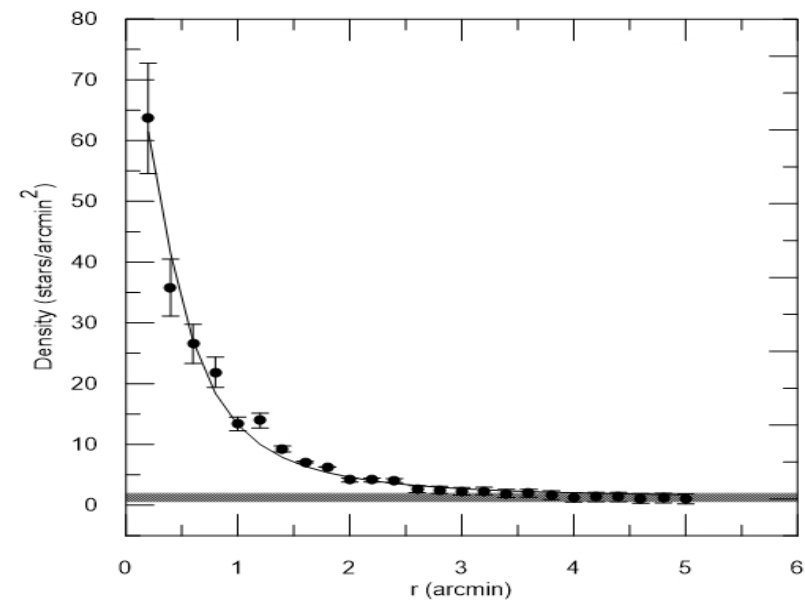

Figure 3. The radial density profile of Be 55. The curved line shows the fitting of King (1966). The vertical bars denote the Poisson errors.

histogram and $\left(G, G_{B P}-G_{R P}\right)$ diagram are presented in the bottom of Fig. 4. The blue and black histograms show 305 and 109 members, respectively. The number of 109 members is in good agreement with the 107 members, classified by Cantat-Gaudin et al. (2018 2020). The applied median Gaia EDR3 parallax ( $\varpi=$ $0.317 \pm 0.059$ mas) on the histogram gives 64 members, and these provide a single stellar sequence (black dots $)$ in the $\left(G, G_{B P}-G_{R P}\right)$ diagram. Since the current Gaia EDR3 parallaxes of the individual stars have a random error, the ensemble median parallax is considered. Accordingly the parallax error is the median of the members. The median astrometric values of 64 members are $\left(\mu_{\alpha}, \mu_{\delta}\right)=(-4.070 \pm 0.078,-4.662 \pm 0.070)$ mas $\mathrm{yr}^{-1}$ and $\varpi=0.317 \pm 0.059$ mas, respectively which are compatible with $\left(\mu_{\alpha}, \mu_{\delta}\right)=(-4.050 \pm 0.219$,$4.618 \pm 0.214) \mathrm{mas} \mathrm{yr}^{-1}$ and $\varpi=0.309 \pm 0.091 \mathrm{mas}$, given by Cantat-Gaudin et al. (2018, 2020).

The determination of the age of $\mathrm{Be} 55$ depends on the memberships of five RSGs/RBGs and two BSs. As was done by L18 (see their figs. 7-10), to querry on their memberships further, their Gaia EDR3 parallaxes against G-mag plus $\mu_{\alpha}$ versus $\mu_{\delta}$ are displayed in Fig. 5(a) and (b). In panel (a) the vertical lines indicate the median Gaia EDR3 parallax $\varpi=0.317 \pm 0.059$ mas (dashed black line), and photometric parallax $\varpi$ $=0.331 \pm 0.043$ from $Q_{V \lambda}-Q^{\prime}$ (orange line, see section 3.4), respectively. As is noticed from panel (a), the parallax uncertainties of five RSGs/RBGs and two BSs (Table 5) almost remain within the error limits of the vertical/horizontal lines. Note that $S 6 / L 145$ 's parallax value is far from the median parallax. But its uncertainty remains the error limit of horizontal dashed line. Here, the notation "L" represents the star ID in L18.

The proper motions of five RSGs/RBGs and two
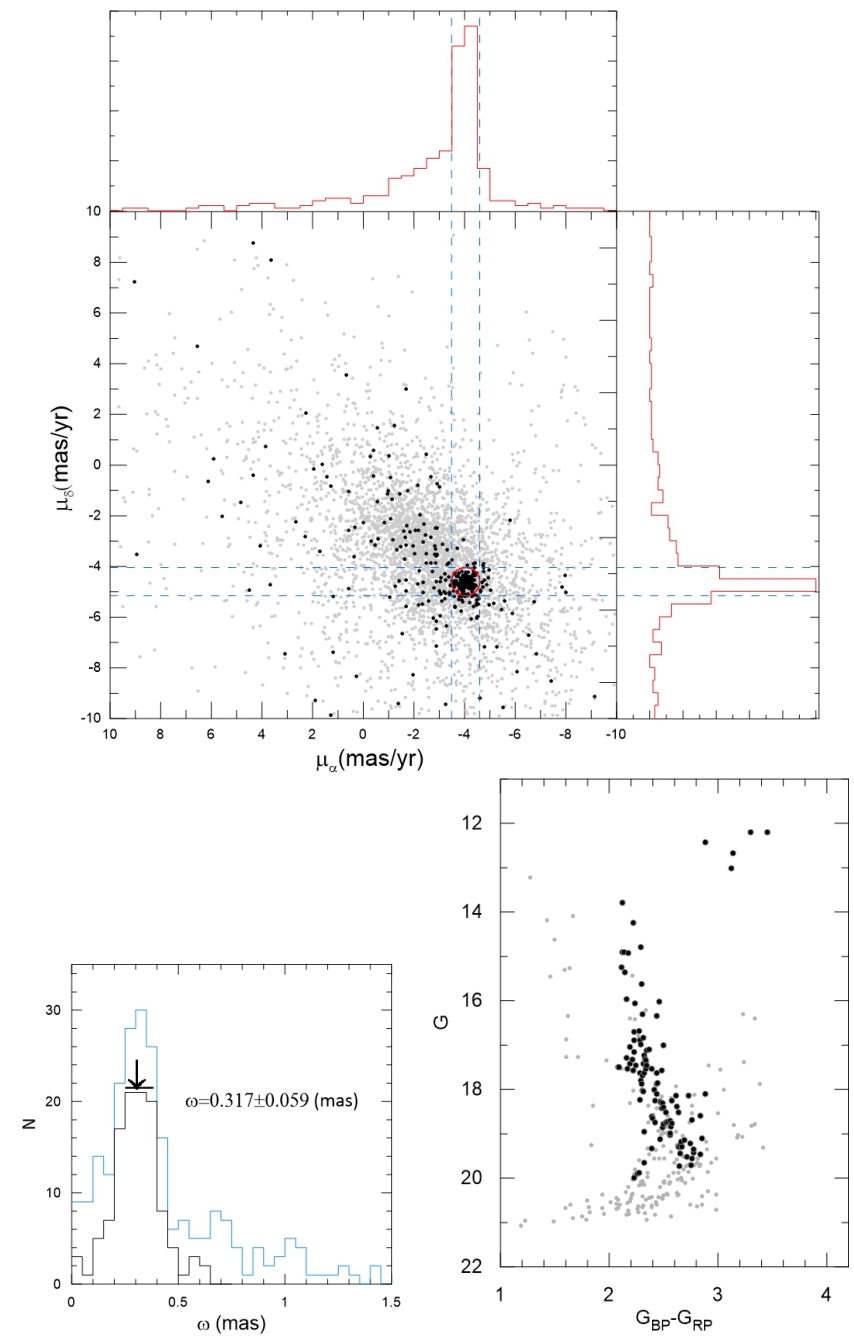

Figure 4. The $\mu_{\alpha}$ versus $\mu_{\delta}$ for 305 stars of Be 55 (filled dots). The field stars inside 15 arcmin are shown with small grey dots. There are probable 109 members in the red circle as the fitted proper motion circle $\left(0.5\right.$ mas $\left.\mathrm{yr}^{-1}\right)$. Gaia EDR3 parallax $(\varpi)$ histogram (left panel) for 305 (blue ) and 109 members (red). On $\left(G, G_{B P}-G_{R P}\right)$, a single stellar cluster sequences of the probable members are clearly visible.

BSs together their errors are inside the borders of the blue circle (panel b). Blue circle is constructed as the following, the derivation of the relation $V_{\tan }=4.74 \mu / \varpi$ provides $\sigma_{V(\tan )}=4.74 \sigma_{\mu} / \varpi$. In this case, it is assumed that the adopted $\varpi$ has no error, and that the velocity of stars in Be 55 is homogeneous and isotropic, $\sigma_{V(\tan )}=\sigma_{V(\mathrm{rad})}$. A20 give the average radial velocity of the bright giants of Be 55 as $V_{\text {rad }}=-27.7 \pm 4.9 \mathrm{~km}$ $\mathrm{s}^{-1}$. Then the expected dispersion in proper motion is obtained as $\sigma_{\mu}=\left(4.9 \mathrm{kms}^{-1}\right) \times \varpi(=0.317 \mathrm{mas}) / 4.74$ $=0.33 \mathrm{mas} \mathrm{yr}^{-1}$. In that case these all seem to be members, for this respect the derived age in this paper is safely interpreted. The global parallax zero point shift, $\Delta \varpi=-0.017$ mas of Gaia EDR3 (Brown et al. , 2020) 

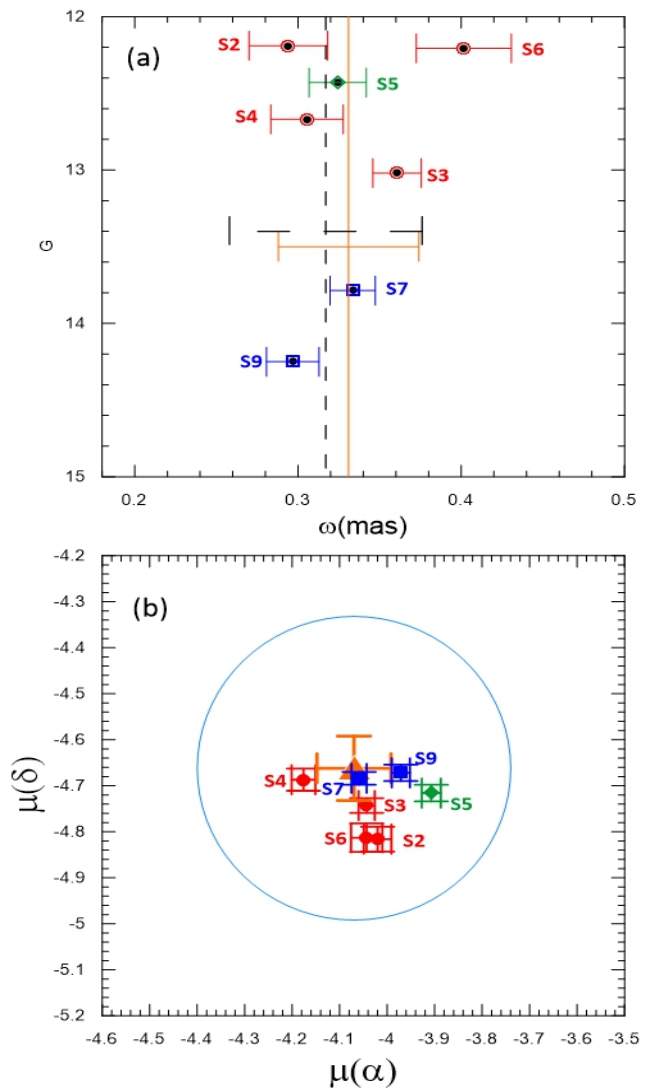

Figure 5. GaiaEDR3 parallax $(\varpi)$ against $G$ mag (panel a) and the $\mu_{\alpha}$ versus $\mu_{\delta}$ (panel b) for five RSGs/RBGs and two BS candidates. The median Gaia EDR3/photometric parallaxes are shown with the vertical dashed black and solid orange lines together their uncertainties (horizontal lines), respectively. The orange triangle of panel (b) denotes the median Gaia EDR3 proper motion components of Be 55. The blue circle represents the expected dispersion (0.33 mas $\left.y r^{-1}\right)$ in proper motion.

is applied to the median value, 0.317 mas, it gives a close distance up to $0.16 \mathrm{pc}$.

The distribution of 64 probable members on the CMDs, $(V, U-B),(V, B-V)$, and $(V, V-I)$ is presented in Fig. 6. Maidanak $U B V I C C D$ observations of the cluster on the CMDs are shown with grey symbols; 89 (panel a), 357 (panel b), 403 (panel c), respectively.

\subsection{Reddening}

The reddening of Be 55 is determined from 38 early type members with $V<16$ mag and $(U-B)<1.1$ on the $(U-B),(B-V)(C C)$ colour-colour plot (Fig. 7). Out of 64 members, 46 members have UBV data. Five members with a red circle are the RSGs/RBGs. The mean reddening from these early type members is estimated as $E(B-V)=1.77 \pm 0.10 \mathrm{mag}$, and thus the red-
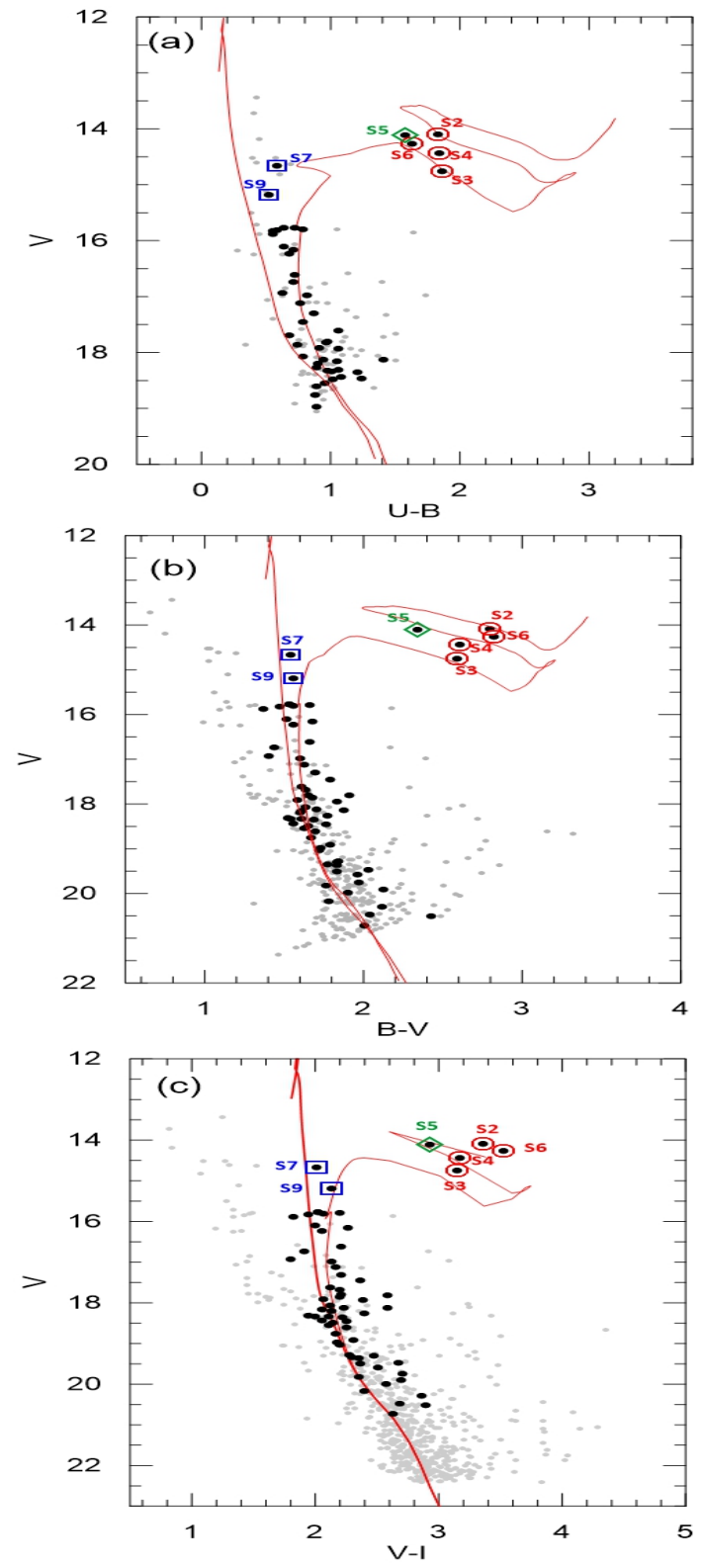

Figure 6. The CMDs of the members; $V-(U-B)(\mathrm{N}=46$, panel a), $V-(B-V)(\mathrm{N}=64$, panel b) and $V-(V-I)(\mathrm{N}=64$, panel c). Solid red lines/curves denote the E12 ZAMS/85 Myr isochrones. Maidanak UBVI CCD observations are shown with grey symbols; 89 (panel a), 357 (panel b), 403 (panel c), respectively. Diamond, filled red circles, and blue squares represent one Cepheid, four RSGs/RBGs and two BSs, respectively. Star designation is from N12.

dened colour sequence of the Schmidt-Kaler (SK82) (Schmidt-Kaler, 1982) (red curve) is fitted to the CC diagram. For the determination of $E(B-V)$, the reddenings $E(V-I), E(V-J), E(V-H)$, and $E\left(V-K_{S}\right)$ have been estimated by using the intrinsic colour relation of early type stars, given by S13 (see their ta- 


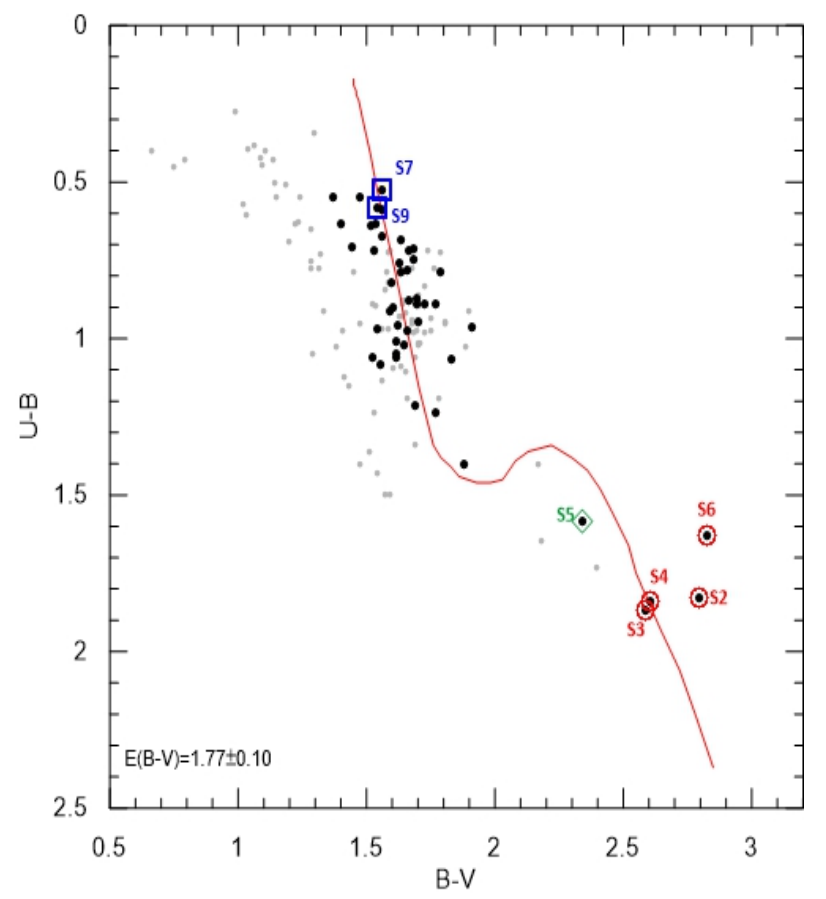

Figure 7. $(U-B),(B-V)(C C)$ diagram for 38 members (filled dots) of Be 55. Grey symbols denote the 97 stars from Maidanak $U B V C C D$ observations. Red curve shows the reddened SK82 main sequence. The symbols of bright evolved stars are the same as Fig. 6.

bles 2-3). Here the colour excess ratio $E(U-B)=$ $0.72 E(B-V)+0.025 E(B-V)^{2}$ of $\mathrm{S} 13$ is adopted. The reddening law of Be 55 using colour excess ratios $E(V-\lambda)$ for $\mathrm{IJHK}_{S}$ photometry has been tested in Fig. 8. 38 members (filled blue dots) lie on the solid line. The total-to-selective extinction ratio is obtained as $R_{V}=3.13 \pm 0.04$ (Table 4), which implies that the reddening law toward Be 55 is quite normal. According to Guetter \& Vrba (1989), colour excess ratio of optical-near infrared colours is related to the total-toselective extinction ratio.

Its highly reddened value, $E(B-V)=1.77 \pm$ $0.10 \mathrm{mag}$ is in agreement with $E(B-V)=1.85 \mathrm{mag}$ of N12, $E(B-V)=1.81 \pm 0.15 \mathrm{mag}$ of $\mathrm{A} 20, E(B-V)=$ $1.75 \mathrm{mag}$ of Molina Lera et al. (2018), $E(B-V)=$ $1.74 \mathrm{mag}$ of L18, and $E(B-V)=1.74 \pm 0.10 \mathrm{mag}$ of Maciejewski \& Niedzielski (2007) within the uncertainties (Table 7), respectively. This reddening is larger than the ones of Tadross (2008) and Bukowiecki et al. (2011). Note that $\mathrm{N} 12$ also give $E\left(J-K_{S}\right)=0.85 \mathrm{mag}$, which converts to $E(B-V)=1.73 \mathrm{mag}$ via a relation $E\left(J-K_{S}\right)=0.49 E(B-V)$ of Dutra et al. (2002).

The reddening value derived from the early type stars cannot be used to deredden late-type supergiants. Therefore, the mean reddening value from their $\left(J-K_{s}\right)$
Table 4. $\mathrm{E}(\mathrm{V}-\lambda) / \mathrm{E}(\mathrm{B}-\mathrm{V})$ ratios (Col. 2) in terms of four colour indices (Col. 1). $\mathrm{R}_{V}$ is the weighted average of four colours. Here $\lambda$ is $\mathbf{I}, \mathbf{J}, \mathrm{H}$ and $\mathbf{K}_{s}$. $\mathrm{N}$ (last column): cluster star numbers.

\begin{tabular}{cll}
\hline Colour & $\mathrm{E}(\mathrm{V}-\lambda) / \mathrm{E}(\mathrm{B}-\mathrm{V})$ & $\mathrm{N}$ \\
\hline V-I & $1.300 \pm 0.050$ & 38 \\
V-J & $2.341 \pm 0.131$ & 38 \\
V-H & $2.659 \pm 0.172$ & 38 \\
V-K $_{s}$ & $2.841 \pm 0.177$ & 38 \\
& $\mathrm{R}_{V}=3.13 \pm 0.04$ & \\
\hline
\end{tabular}

colours of the five evolved stars (Table 5) is estimated as $E\left(J-K_{s}\right)=0.92 \pm 0.06 \mathrm{mag}$, by utilising the intrinsic colour, $\left(J-K_{s}\right)_{0}$ of Koornneef (1983). This converts to $E(B-V)=1.84 \pm 0.11 \mathrm{mag}$ from the relation $E\left(J-K_{s}\right)=0.49 E(B-V)$ (Dutra et al., 2002). Their reddenings are listed in Col. 5 of Table 5. For Be 55, A20 mention a non-negligible differential reddening. The reddened values $E(B-V)=1.77 \pm 0.10 \mathrm{mag}$ (early type stars) and $E(B-V)=1.84 \pm 0.11 \mathrm{mag}$ (five evolved stars) imply a differential reddening in Be 55. The members selected in Fig. 7 are scattered over more than $0.3 \mathrm{mag}$ in $(B-V)$ for a given $(U-B)$. Likewise, the members are scattered over more than 0.5 mag in $\left(G, G_{B P}-G_{R P}\right)$ (Fig. 4).

The spatial variation of reddening of $\mathrm{Be} 55$ as the reddening map, is derived from its member stars (red dots) and field stars (black dots), and is presented in Fig. 9. The size of the dots is scaled to the magnitude of the star. The coloured line types as iso-reddening contours represent different amounts of reddening $E(B-V)$. From Fig. 9, there seems to be a differential reddening across the cluster due to a slight variation of $E(B-V)$. Most young OCs with ages younger than $10 \mathrm{Myr}$ show a differential reddening across the field of view. But for slightly old OCs, it is not well known whether there is a differential reddening across the cluster or not. That's the reason to check the differential reddening across the cluster.

\subsection{Distance}

Instead of the reddening-corrected CMDs, distance modulus/distance of $\mathrm{Be} 55$ is obtained by using the ZAMS fitting method with the reddening-independent

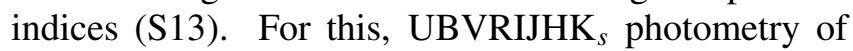
38 early type stars is used. The reddening-independent quantities, $Q^{\prime}, Q_{V I}, Q_{V J}, Q_{V H}$ and $Q_{V K_{S}}$ are utilised. The $Q_{V \lambda}-Q^{\prime}$ plots for 38 early type members have been displayed in Fig. 10. Solid lines denote the fitted ZAMS relation of Sung et al. (2013) to the members. As sug- 

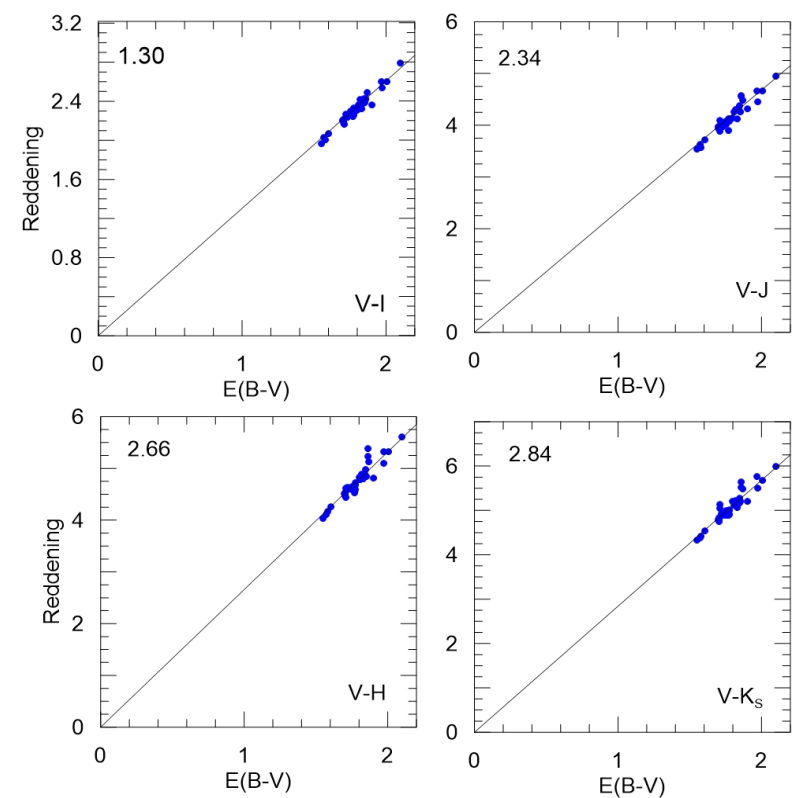

Figure 8. $E(V-\lambda)$ versus $\mathrm{E}(\mathrm{B}-\mathrm{V})$ relations. $\lambda$ denotes $\mathrm{IJHK}_{S}$ photometry.The solid line means $R_{V}=3.1$. The colour excess ratios from the $I J K_{S}$ data consistently show that the reddening law toward Be 55 is normal.

gested by Lim et al. (2014), ZAMS should be fitted to the lower ridge of the MS band to avoid the effects of multiplicity and evolution. The ZAMS line is shifted up and down in Fig. 10 by 0.1 mag. The error of this method is about 0.20 mag. Once the ZAMS has been adjusted above and below the distribution of the members (Fig. 10), the distance modulus from four colour indices is obtained as $\left(V_{0}-M_{V}\right)=12.40 \pm 0.20 \mathrm{mag}$, equivalent to $3.02 \pm 0.28 \mathrm{kpc}$, which are adopted for this paper. The median Gaia EDR3 parallax is $\varpi=$ $0.317 \pm 0.059$ mas, which corresponds to $\left(V_{0}-M_{V}\right)=$ $12.49 \pm 0.41 \mathrm{mag}(\mathrm{d}=3.15 \pm 0.59 \mathrm{kpc})$. The two distances

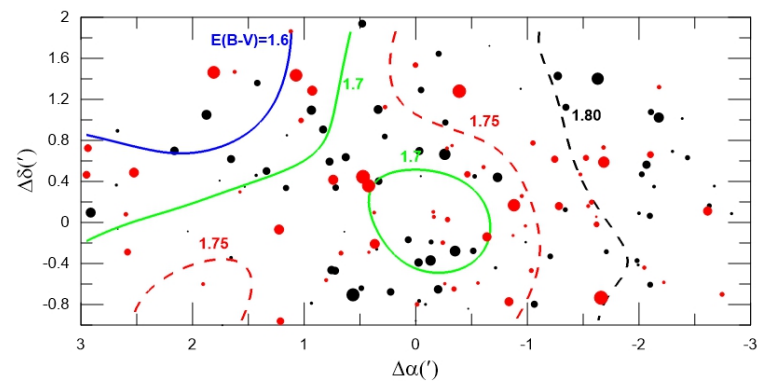

Figure 9. Reddening map of Be 55. Red and black dots shows the member and the field stars, respectively. The size of the dots is scaled to the magnitude of the star. The coloured line types as iso-reddening contours represent different amounts of reddening $E(B-V)$.
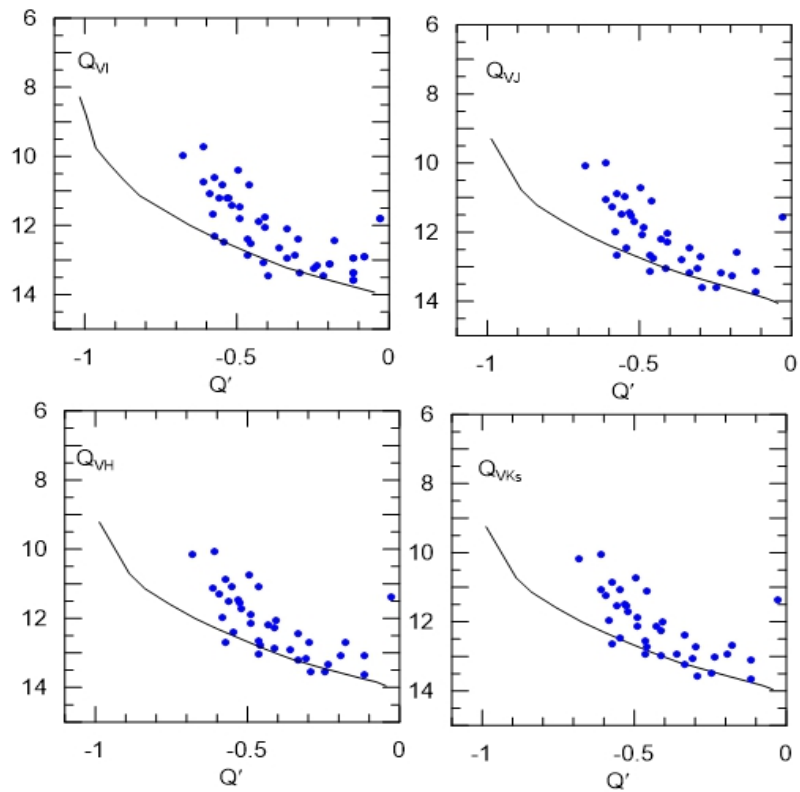

Figure 10. $Q_{V \lambda}$ versus $Q^{\prime}$ diagrams (38 early type members, filled blue dots) for determination of distance modulus of Be 55. $\lambda$ denotes $\mathrm{IJHK}_{S}$ filters. A careful ZAMS fitting to the lower boundary of the MS band was carried out in the reddening-independent $Q_{V \lambda}-Q^{\prime}$ planes. The ZAMS relation of $\mathrm{S} 13$ is used to determine the distance to the cluster after adjusting by $12.4 \pm 0.2$, respectively.

are compatible within the uncertainties.

\section{Age of Be 55}

$M_{b o l}-\log T_{e f f}$ diagram (HRD) for 46 members (41 early type and five RSGs/RBGs) is presented in Fig. 11. Their effective temperature and $B C$ values have been determined from their $(U-B)_{0}$ and $(B-V)_{0}$ by utilising table 5 of S13. Their $M_{b o l}$ values are obtained from the relation $M_{b o l}=B C+V-R_{V} E(B-V)-\left(V_{0}-M_{V}\right)$. For this, the UBV photometry of 41 early type and five RSGs/RBGs is de-reddened by $E(B-V)=1.77 \mathrm{mag}$ and $E(B-V)=1.84 \mathrm{mag}$, respectively.

The metal abundance, $[M / H]=0.07 \pm 0.12(Z=$ 0.014 ) of Be 55 , given by L18 is considered for selecting the isochrone. Note that $\mathrm{Be} 55$ has a extended main sequence. Due to the diversity of the rotational velocity even in a cluster (Lim et al. 2019), MS turn-off broadens. Broadening in the main sequence of the CMD could be also due to binarity, peculiarity, rotation in the stars. Therefore, the solar abundance Geneva models of Ekström et al. (2012) (hereafter E12) $\left(V / V_{\text {crit }}=0.4\right)$ and Georgy et al. (2013) (hereafter G13) (for B-type stars and $V / V_{\text {crit }}=0.3$ ) have been fitted to the members in the $M_{b o l}-\log T_{e f f}$ (Fig. 11) and de-reddened $\left(V_{0}, B-\right.$ 
Table 5. Properties of seven bright stars in Be 55.

\begin{tabular}{lccccccccrr}
\hline $\begin{array}{l}\text { Star no } \\
(\mathrm{N} 12 / \mathrm{L} 18)\end{array}$ & $\begin{array}{c}\mathrm{V} \\
\mathrm{mag}\end{array}$ & $(\mathrm{B}-\mathrm{V})$ & $(\mathrm{U}-\mathrm{B})$ & $\mathrm{E}\left(\mathrm{J}-\mathrm{K}_{S}\right) / \mathrm{E}(\mathrm{B}-\mathrm{V})$ & $m\left(M_{\odot}\right)$ & $\begin{array}{c}\mathrm{M}_{V} \\
\mathrm{mag}\end{array}$ & $\begin{array}{c}\varpi \\
\mathrm{mas}\end{array}$ & $\begin{array}{c}d_{\text {Gaia }} \\
\mathrm{kpc}\end{array}$ & $\begin{array}{c}d_{p h .} \\
\mathrm{kpc}\end{array}$ & $\begin{array}{r}\text { SpT } \\
(\mathrm{A} 20)\end{array}$ \\
\hline$S 5 / L 107$ & 14.106 & 2.340 & 1.582 & $0.863 / 1.726$ & 5.9 & -3.99 & $0.324 \pm 0.017$ & $3.09 \pm 0.16$ & $3.47 \pm 0.45$ & F8Ib \\
$S 3 / L 163$ & 14.754 & 2.588 & 1.866 & $0.933 / 1.866$ & 5.8 & -3.35 & $0.360 \pm 0.015$ & $2.78 \pm 0.12$ & $2.84 \pm 0.37$ & G8II \\
$S 4 / L 110$ & 14.437 & 2.604 & 1.840 & $0.885 / 1.770$ & 5.9 & -3.67 & $0.305 \pm 0.022$ & $3.28 \pm 0.24$ & $3.26 \pm 0.42$ & K0Ib-II \\
$S 2 / L 196$ & 14.094 & 2.796 & 1.826 & $1.006 / 2.012$ & 6.0 & -4.01 & $0.294 \pm 0.024$ & $3.40 \pm 0.28$ & $2.30 \pm 0.30$ & K0Ib \\
$S 6 / L 145$ & 14.266 & 2.825 & 1.628 & $0.906 / 1.812$ & 6.0 & -3.84 & $0.402 \pm 0.029$ & $2.49 \pm 0.18$ & $3.07 \pm 0.40$ & K4II \\
$S 9 / L 198$ & 15.186 & 1.562 & 0.524 & $-/ 1.770$ & 7.5 & -2.70 & $0.297 \pm 0.016$ & $3.37 \pm 0.18$ & $2.95 \pm 0.38$ & B3-4IIIShell \\
$S 7 / L 94$ & 14.666 & 1.543 & 0.581 & $-/ 1.770$ & 7.5 & -3.22 & $0.334 \pm 0.014$ & $2.99 \pm 0.13$ & $2.95 \pm 0.38$ & B4IV \\
\hline
\end{tabular}

$\left.V_{0}\right)$ (Fig. 12). $\left(V_{0}-M_{V}\right)=12.40 \pm 0.20 \mathrm{mag}(\mathrm{Q}$ technique) and the reddenings $E(B-V)=1.77 \pm 0.10 \mathrm{mag}$ (for early type stars) and $E(B-V)=1.84 \pm 0.11 \mathrm{mag}$ (for evolved stars) have been applied to the E12 and G13 isochrones while fitting.

As is seen in Fig. 11, the $85 \pm 13$ Myr E12 isochrone with high rotation (blue curve) describes general morphology of HRD without accurate locations. The 85 Myr E12 isochrone (blue curve) is almost consistent with the RSGs/RBGs, $S 4 / L 110, S 5 / L 107$ (Cepheid), and $S 6 / L 145$, except for $S 3 / L 163$. Also, the $70 \pm 10$ Myr G13 isochrone (red curve) with moderate rotation for B-type stars provides good fit with the RSGs/RBGs, $S 2 / L 196, S 4 / L 110$ and $S 5 / L 107$ (Cepheid), except for $S 3 / L 163$ and $S 6 / L 145$. Note that $S 5 / L 107$ appears to be on the core-He burning blue loop. Note that nonrotating E12 isochrone (blue dashed line) gives a faint turn-off, and going below the RSGs/RBGs.

On the $V_{0}-(B-V)_{0}$ (Fig. 12), S2/L196 and three RSGs/RBGs occupy positions which are consistent with the 85 Myr E12 isochrone. However, the 70 Myr G13 isochrone is not compatible with the positions of the RSGs/RBGs.

The two BSs, $S 9 / L 198$ and $S 7 / L 94$ locate between ZAMS and the $45 \mathrm{Myr}$ isochrone (Figs. 11-12). The age of BSs seems to be definitely younger than the cluster. From Figs. 11-12, there appears an extended mainsequence turn-off (eMSTO), since the main sequence band is expected to broaden in CMDs due to their different main sequence lifetimes. Also, the broadening in the MS band may also be due to differential reddening across the cluster because the scatter in $E(B-V)$ is about $0.1 \mathrm{mag}$ (section 3.3). One can expect about 0.3 mag scatter $(3 \sigma)$. since the width of MS band is about 0.3 mag.

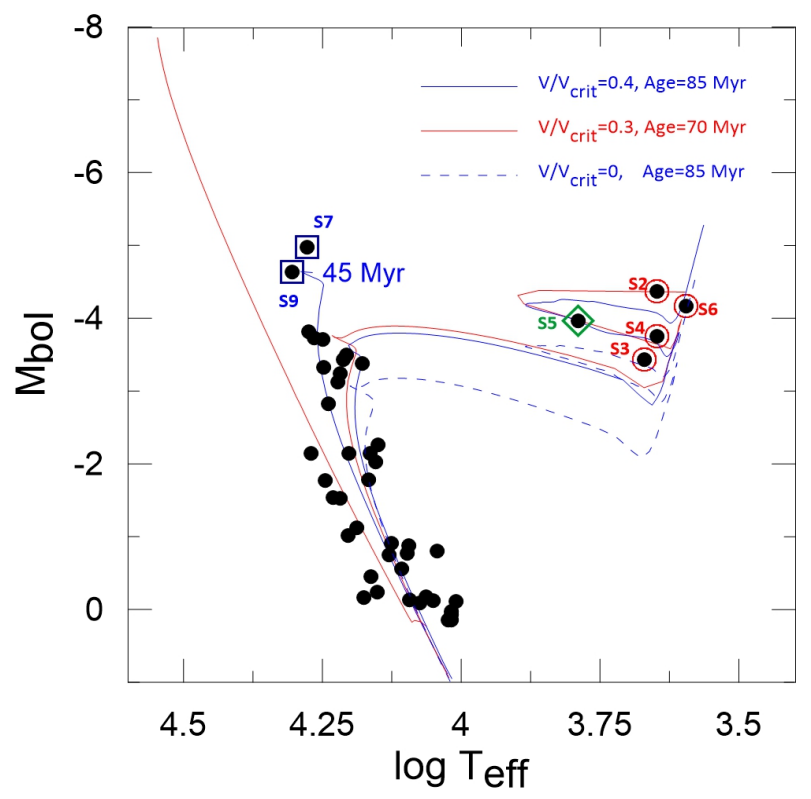

Figure 11. $M_{b o l}$ versus $\log T_{e f f}$ of 46 probable members. Solid and dashed blue curves represent rotating/non-rotating E12 isochrones. Red curve shows G13 isochrone for B type stars with $V / V_{\text {crit }}=0.3$. The solid red line represents the ZAMS of E12. The symbols are the same as Fig. 6.

\section{Kinematics and orbital parameters}

From the radial velocities of A20 and the Gaia EDR3 proper motions for five RSGs/RBGs, their heliocentric velocities $(U, V, W)$ in the right-hand system have been calculated from the algorithm of Johnson and Soderblom (1987). The photometric distance of Be 55 $(3.02 \pm 0.28 \mathrm{kpc})$ is adopted, instead of their individual parallaxes. These space velocities are transformed to the components $U^{\prime}, V^{\prime}, W^{\prime}$ by correcting for the Solar motion $(U, V, W)_{\odot}=(+11.10,+12.24,+7.25) \mathrm{km}$ $\mathrm{s}^{-1}$ with respect to the local standard of rest (LSR) Schönrich and Binney (2010). Here, $\mathrm{R}_{\odot}=8.2 \pm 0.1 \mathrm{kpc}$ 


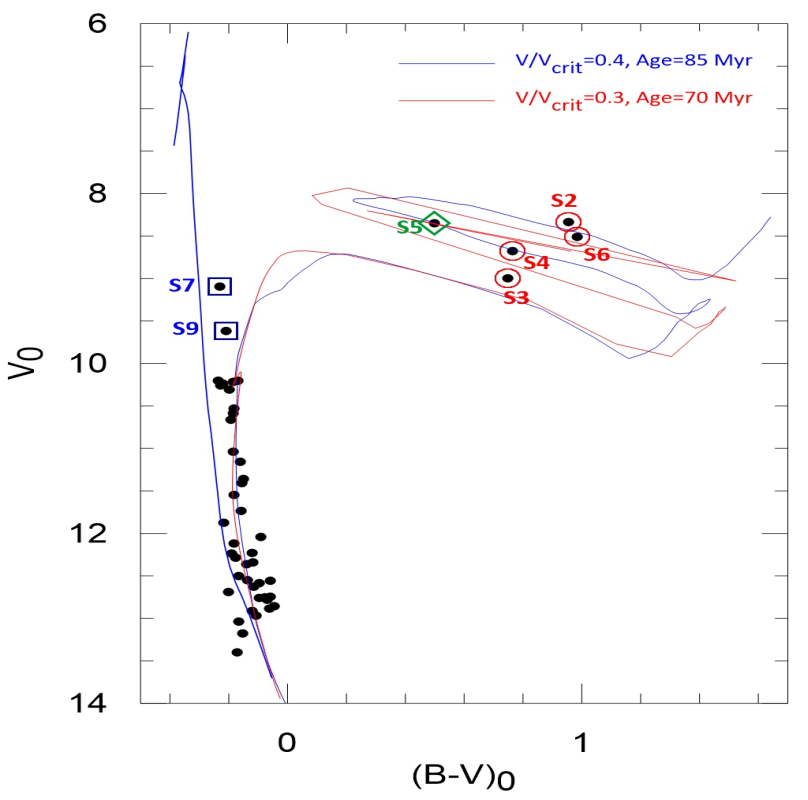

Figure 12. $V_{0}-(B-V)_{0}$ of 46 probable members. The symbols are the same as Fig. 11.
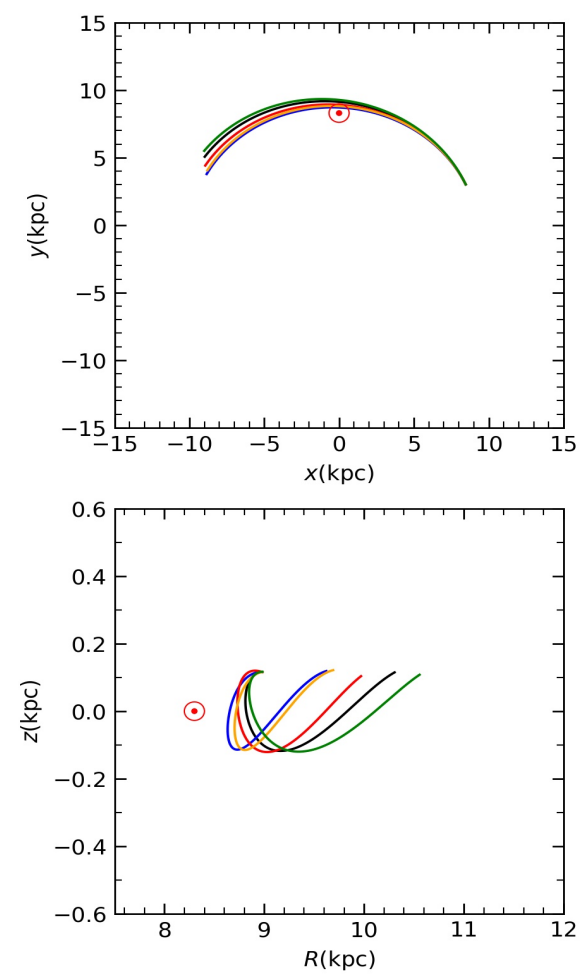

Figure 13. The orbits of five RSGs/RBGs on $x-y$ (kpc) (top panel) and $\mathrm{z}-\mathrm{R}(\mathrm{kpc})$ (bottom panel). Large circle shows the position of the Sun, $\left(z_{\odot}, R_{\odot}\right)=(0,8.2 \mathrm{kpc})$.

(Bland-Hawthorn et al. 2016) and $V_{L S R}=239 \mathrm{~km} \mathrm{~s}^{-1}$
(Brunthaler et al., 2011) are adopted. The heliocentric cartesian distances $\left(x^{\prime}, y^{\prime}, z^{\prime}\right)(\mathrm{kpc})$ and LRS-velocity components $\left(U^{\prime}, V^{\prime}, W^{\prime}\right)$ have been transformed to Galactic Rest of Frame (GSR) i.e., $(x, y, z)(\mathrm{kpc})$ and $\left(V_{x}, V_{y}, V_{z}\right)$ from the equations of Kepley et al. (2007). The Galactocentric velocity component $\left(V_{\Phi}\right)\left(\mathrm{km} \mathrm{s}^{-1}\right)$ (or azimuthal velocity) in a cylindrical frame is estimated via $V_{\Phi}=\frac{x V_{y}-y V_{x}}{R}$. Here, $V_{\Phi}<0$ means prograde. By utilising the "MWPotential2014" code in the galpy-code library 11 written by Bovy (2015), peri- and apo-galactic distances $\left(R_{\min }, R_{\max }\right)(\mathrm{kpc})$ and the maximum height distance $\left(\mathrm{z}_{\max }\right)(\mathrm{kpc})$ have been obtained. The orbital eccentricity (ecc) is estimated via the relation $e=\left(R_{\max }-R_{\min }\right) /\left(R_{\max }+R_{\min }\right)$. Five evolved member's orbits have been integrated for $85 \mathrm{Myr}$ within the Galactic potential. The galactic potential as sum of the Galactic components is explained by Bovy (2015). Their orbital angular momentum components $J_{x}, J_{y}, J_{z}$ and $J_{\perp}\left(\mathrm{kpc} \mathrm{km} \mathrm{s}^{-1}\right)$ are calculated from the equations of Kepley et al. (2007). The total angular momentum $\mathrm{J}_{\perp}$ is defined as $J_{\perp}=\left(J_{x}^{2}+J_{y}^{2}\right)^{1 / 2}$. The relations of $\mathrm{x}-\mathrm{y}(\mathrm{kpc})$ and $\mathrm{z}-\mathrm{R}(\mathrm{kpc})$ of the five RSGs/RBGs are shown in Fig. 13.

Table 6. For the available Gaia EDR3 proper motion components (mas $\mathrm{yr}^{-1}$ ) and spectroscopic radial velocity data $\left(\mathrm{km} \mathrm{s}^{-1}\right)$ of five RSGs/RBGs, kinematics $\left(U, V, W, V_{\Phi}\right) \mathrm{km}$ $\mathrm{s}^{-1}$, orbital parameters $\left(\mathrm{R}_{\max }, \mathrm{R}_{\min }, \mathrm{Z}_{\max }\right)(\mathrm{kpc})$, eccentricity (ecc), and angular momentums $\left(J_{z}\right.$ and $\left.J_{\perp}\right)\left(\mathrm{kpc} \mathrm{km} \mathrm{s}^{-1}\right)$, respectively.

\begin{tabular}{|c|c|c|c|c|}
\hline Star & $\mu_{\alpha}$ & $\mu_{\delta}$ & $V_{\text {rad }}$ & \\
\hline S2 & $-4.020 \pm 0.029$ & $-4.816 \pm 0.027$ & $-24.55 \pm 0.39$ & \\
\hline S3 & $-4.043 \pm 0.017$ & $-4.743 \pm 0.016$ & $-33.33 \pm 0.47$ & \\
\hline S4 & $-4.176 \pm 0.025$ & $-4.687 \pm 0.024$ & $-28.97 \pm 0.47$ & \\
\hline S5 & $-3.907 \pm 0.020$ & $-4.716 \pm 0.018$ & $-31.63 \pm 0.86$ & \\
\hline \multirow[t]{2}{*}{ S6 } & $-4.043 \pm 0.033$ & $-4.813 \pm 0.031$ & $-21.61 \pm 0.60$ & \\
\hline & $\mathrm{U}$ & $\mathrm{V}$ & W & $V_{\Phi}$ \\
\hline S2 & 90.71 & -19.59 & -7.55 & -252.38 \\
\hline S3 & 90.66 & -28.39 & -6.88 & -244.08 \\
\hline S4 & 91.18 & -24.04 & -4.84 & -248.35 \\
\hline S5 & 88.94 & -26.72 & -7.97 & -245.07 \\
\hline \multirow[t]{2}{*}{ S6 } & 90.76 & -16.60 & -7.23 & -255.22 \\
\hline & $\mathrm{R}_{\min }$ & $\mathrm{R}_{\max }$ & ecc & \\
\hline S2 & 8.81 & 10.31 & 0.08 & \\
\hline S3 & 8.63 & 9.62 & 0.05 & \\
\hline S4 & 8.73 & 9.97 & 0.07 & \\
\hline S5 & 8.70 & 9.69 & 0.05 & \\
\hline \multirow[t]{2}{*}{ S6 } & 8.85 & 10.56 & 0.09 & \\
\hline & $\mathrm{z}_{\max }$ & $J_{z}$ & $J_{\perp}$ & \\
\hline S2 & 0.12 & -2266 & 24 & \\
\hline S3 & 0.12 & -2192 & 24 & \\
\hline S4 & 0.12 & -2230 & 33 & \\
\hline S5 & 0.12 & -2200 & 24 & \\
\hline S6 & 0.13 & -2292 & 24 & \\
\hline
\end{tabular}

$\overline{{ }^{1} \text { http://github.com/jobovy/galpy }}$ 
Table 7. Comparison with the literature for Be 55.

\begin{tabular}{|c|c|c|c|c|c|c|c|c|}
\hline $\begin{array}{c}\mathrm{E}(\mathrm{B}-\mathrm{V}) \\
\mathrm{mag}\end{array}$ & $\begin{array}{c}\left(V_{0}-M_{V}\right) \\
\mathrm{mag}\end{array}$ & $\begin{array}{l}d(\mathrm{kpc}) \\
\mathrm{kpc}\end{array}$ & $Z$ & $\log A g e$ & $\begin{array}{l}\text { Age } \\
\text { Myr }\end{array}$ & Isochrone & Photometry & Ref. \\
\hline $1.77 \pm 0.10$ & $12.40 \pm 0.20$ & $3.02 \pm 0.28$ & 0.014 & $7.93 \pm 0.06$ & $85 \pm 13$ & Ekström et al. 2012) & CCD $U B V I$ & This paper \\
\hline $1.74 \pm 0.07$ & $11.71 \pm 0.30$ & $2.20 \pm 0.30$ & & 7.80 & $63 \pm 12$ & PLR of S5 Cepheid & CCD $V R$ & Lohr et al. 2018) \\
\hline $1.85 \pm 0.16$ & $13.0 \pm 0.30$ & $3.98 \pm 0.55$ & solar & 7.70 & $50 \pm 10$ & Marigo et al. 2008) & $\mathrm{CCD} U B V$ & Negueruela \& Marco 2012) \\
\hline $1.81 \pm 0.15$ & $12.55 \pm 0.15$ & $3.24 \pm 0.22$ & solar & $7.80 \pm 0.10$ & $63 \pm 15$ & Ekström et al. 2012) & $\operatorname{CCD} U B V$ & \begin{tabular}{|ll} 
Alonso-Santiago et al. 2020) & (20) \\
\end{tabular} \\
\hline 1.75 & 12.40 & 3.02 & solar & $7.50-8.50$ & $30-100$ & Bressan et al. 2012) & $u g r$ & Molina Lera et al. 2018) \\
\hline $1.74 \pm 0.10$ & 10.42 & $1.21 \pm 0.31$ & solar & 8.50 & 315 & Bertelli 1994$)$ & $\mathrm{CCD} B V$ & Maciejewski \& Niedzielski 2007) \\
\hline 1.50 & & $1.44 \pm 0.07$ & solar & 8.48 & 300 & Bonatto et al. 2004) & $2 M A S S-J H K_{S}$ & Tadross 2008) \\
\hline 1.15 & 12.23 & $1.70 \pm 0.13$ & 0.019 & 8.95 & 891 & Girardi et al. 2002) & $2 M A S S-J K_{S}$ & Bukowiecki et al. 2011) \\
\hline $1.55\left(A_{V}=4.8\right)$ & 12.20 & 2.75 & 0.015 & 8.30 & 200 & Bressan et al. 2012) & Gaia DR2 & Cantat-Gaudin et al. 2018, 2020) \\
\hline
\end{tabular}

Table 8. Distances for the values of $V, M_{V}, P=5.85$ day, and $E(B-V)$ of Cepheid $S 5$. The methods and their references for $M_{V}$ are listed in Cols.5-6.

\begin{tabular}{ccccccc}
\hline $\mathrm{d}$ & $\mathrm{V}$ & $M_{V}$ & $E(B-V)$ & Methods for $M_{V}$ & Ref. & Remarks \\
$\mathrm{kpc}$ & $\mathrm{mag}$ & $\mathrm{mag}$ & $\mathrm{mag}$ & & & \\
\hline $3.47 \pm 0.45$ & 14.106 & -3.99 & 1.73 & E12 isochrone & 1 & This paper \\
$2.78 \pm 0.32$ & 14.106 & -3.52 & 1.73 & $-(2.67 \pm 0.16)(\log \mathrm{P}-1)-(4.14 \pm 0.05)$ & 2 & This paper \\
$3.09 \pm 0.16$ & & & & & & Gaia EDR3 \\
$2.20 \pm 0.30$ & 13.834 & -3.23 & 1.74 & $-(2.88 \pm 0.18) \log \mathrm{P}-(1.02 \pm 0.16)$ & 3 & $\mathrm{~L} 18$ \\
$2.40 \pm 0.30$ & 13.834 & -3.93 & 1.74 & $-(2.43 \pm 0.12)(\operatorname{logP}-1)-(4.5 \pm 0.02)$ & 4 & $\mathrm{~L} 18$ \\
$3.03 \pm 0.37$ & 13.834 & & 1.81 & $\mathrm{a}(\operatorname{logP}-1)+\mathrm{b}$, for JHK & 5 & $\mathrm{~A} 20$ \\
\hline
\end{tabular}

Table Notes. [1] Ekström et al. [2012], [2]: Lazovik and Rastorguev [2020], [3]: Anderson et al. [2013, [4] Benedict et al. [2007], [5] Chen et al. 2017],
[2],

\section{Discussion and Conclusion}

The photometric distance modulus/distance of Be 55 as $\left(V_{0}-M_{V}\right)=12.40 \pm 0.20 \mathrm{mag}(3.02 \pm 0.28 \mathrm{kpc})$ is better and well consistent with the median Gaia EDR3 distance $(3.15 \pm 0.59 \mathrm{kpc})$. These distances locate Be 55 near the Perseus Spiral arm. The photometric distance of this paper is in concordance with $3.24 \pm 0.22 \mathrm{kpc}$ of A20 and $3.02 \mathrm{kpc}$ of Molina Lera et al. (2018) (Table 7). It is rather smaller than N12 but is farther than L18. L18'distance is from period-luminosity relation (PLR) of Cepheid $S 5$ (Table 8). N12 obtain its distance modulus from the dereddened ZAMS of Mermilliod (1981) and SK82 as $13.0 \pm 0.30 \mathrm{mag}$ on $M_{V}-(B-V)$. This corresponds to a distance, $3.98 \pm 0.55 \mathrm{kpc}$. L18'distance locates Be 55 on the outer edge of Local arm rather than in the Perseus arm as N12 suggest. A mismatch between the distances of L18 and N12 is explained by the underestimated uncertainties in N12's distance modulus. The other literature give close distances.

The distance $2.78 \mathrm{kpc}$ of Cepheid $S 5 / L 107$, from the PLR of Lazovik and Rastorguev (2020) is consistent with the ones of Gaia EDR3 and A20 within the errors (Table 8). Note that L18 give close distances. $M_{V}=-3.99$ from E12 isochrone estimates a large distance, $3.47 \mathrm{kpc}$.

The E12 isochrone (high rotation) fittings to
HRD/CMD derive turn-off age, $85 \pm 13 \mathrm{Myr}$ of Be 55, by taking care five RSGs/RBGs. For this age, the masses of five RSGs/RBGs from the E12 isochrone are about $6 M_{\odot}($ Col. 6 of Table 5). The 70 Myr G13 isochrone with moderate rotation does not provide a good fit to the RSGs/RBGs on the $V_{0}-(B-V)_{0}$ (Fig.12). The age $85 \mathrm{Myr}$ is somewhat older than N12, L18, and A20 (Table 7) but falls in the range of 30-100 Myr of Molina Lera et al. (2018). From the non-rotating PARSEC isochrones, A20 give its age as $63 \pm 15 \mathrm{Myr}$ (see their fig. 6 and table 1), by considering evolved members. The age $85 \mathrm{Myr}$ also falls in the range of 63-105 Myr, which is found from the period-age relations in table 14 of A20 for Cepheid S5/\#107. Note that N12 apply two isochrones of Marigo et al. (2008); $\log t=7.6(40 \mathrm{Myr})$ and $\log t=7.7$ (50 Myr) on $(V, U-B)$. By taking $E\left(J-K_{S}\right)=0.85 \mathrm{mag}, \mathrm{N} 12$ also apply the same isochrones to $\left(K_{S}, J-K_{S}\right)$, and give an age $50 \mathrm{Myr}$. The other literature values find more old ages (Table 7), than this paper.

From Table 7, Note that Cantat-Gaudin et al. (2018, 2020) from Gaia DR2 photometry give a less reddening, a close distance and an old age, $200 \mathrm{Myr}$, as compared to the values of this paper.

The spread of the brightness of the RSGs/RBGs in the HRD/CMDs indicates a much large spread in age. This feature may be the result of the diversity of stellar rotation among evolved cluster members (Lim et al. , 2019; Lim et al., 2016; Sung et al., 1997), which indicate the necessity of cluster isochrone with nonsingle rotational velocity distribution. The stars in OCs do not have the same rotational velocity (Lim et al. 2019). Some stars have very low rotational velocities, and some may be fast rotators. For that case, even the same mass stars are not the same position in the HRD because fast rotators have longer MS lifetime. This feature also indicates the necessity of cluster isochrones with non-single rotational velocity distribution. As a result, the possible inconsistences on the locations of the RSGs/RBGs to the rotating/non-rotating isochrones may be resulting from the age spread of stars in young 
OCs. According to A20, the possible inconsistence of the locations of the RSGs/RBGs on HRD/CMD is due to the strong reddening, rather than metal abundance.

Two BS candidates in $V_{0}-(B-V)_{0}$ (Fig. 12) lie on $\left(V_{0}<10.01 \mathrm{mag}\right)(V<15.50 \mathrm{mag})$ and $\left((B-V)_{0}<\right.$ $0.03)(B-V)<1.80$. These limits for $V_{0}$ and $(B-$ $V)_{0}$ which BSs occupy in CMDs are similar to those given by Carney (2001, fig. 19) and Carraro et al. (2010, fig. 10). The positions of two BSs locate between ZAMS and the $85 \mathrm{Myr}$ isochrone. The age of $\mathrm{BSs}$ is definitely younger than the cluster. Therefore the 45 Myr isochrone is drawn up to MS turn-off. As discussed by Ferraro (2016), BSs are commonly defined as stars brighter and bluer than the main-sequence (MS) turnoff in open/globular clusters. Therefore, their origin cannot be explained with normal single star evolution. Two main formation mechanisms are proposed: (1) mass transfer in binary systems (McCrea , 1964) possibly up to the complete coalescence of the two stars, and (2) stellar collisions (Hills and Day, 1976). Both these processes can potentially bring new hydrogen into the core and therefore 'rejuvenate' a star to its MS stage (Lombardi et al. , 2002, Chen and Han 2009). According to Sandage (1953) and Tout et al. (1997), the increase in mass of a star makes it look younger than it is.

The kinematics, orbital and angular momentum values of the five evolved members (Table 6) indicate that $\mathrm{Be} 55$ is a member of Galactic thin disk population, which is also consistent with its metallicity, $[M / H]=$ $0.07 \pm 0.12$. With the circular orbits, ecc $=[0.05,0.09]$, Be 55 does not seem to be completed a tour around the center of the Galaxy (top panel of Fig. 13). They reach to $\mathrm{z} \sim 0.13 \mathrm{kpc}$, and their birth places are at $\sim$ $9 \mathrm{kpc}$ (bottom panel of Fig. 13). However, their orbits show that the cluster passed a part of its time at $R_{\text {min }}=8.63-8.85 \mathrm{kpc}$.

The total mass for 64 early type members is obtained as $M_{t o t}=224 M_{\odot}$ from $85 \mathrm{Myr}$ E12 isochrone. From the photometric distance $(3.02 \pm 0.28 \mathrm{kpc})$ and angular size $\theta \sim 0.073 \mathrm{deg}(0.0013 \mathrm{in} \mathrm{rad})$, the diameter of Be 55 is determined as $3.85 \mathrm{pc}$. In order to check its stability, the maximum tangential velocity of the mem-

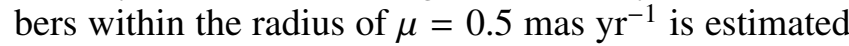
as $7.2 \mathrm{~km} \mathrm{~s}^{-1}$, via the relation, $V_{\text {tan }}=4.74 \mu \times d(k p c)$. These values indicate that its virial mass is about $M_{v i r}=$ $25100 M_{\odot}$, which is far larger than the cluster mass, $224 M_{\odot}$. Be 55 with its total mass, $224 M_{\odot}$, depending on its location of $R_{G C}=9.04 \mathrm{kpc}$ and $\ell=93^{\circ} .03$ is a survivor against internal and external perturbations related to, e.g. stellar evolution, mass segregation, spiral arms, and encounters with the disk and giant molecular clouds.

\section{Acknowledgements}

I thank H. Sung for providing his private photometric data, and the interpretations on the analysis. Y. Karatas and $\mathrm{H}$. Cakmak are also thanked for the kinematics and dynamics. The referee is thanked for the useful suggestions. This paper has made use of results from the European Space Agency (ESA) space mission Gaia, the data from which were processed by the Gaia Data Processing and Analysis Consortium (DPAC). Funding for the DPAC has been provided by national institutions, in particular the institutions participating in the Gaia Multilateral Agreement. The Gaia mission website is http: //www.cosmos.esa.int/gaia.

\section{References}

Akkaya Oralhan, Í., Michel, R., Schuster, W.J, Karataş, Y., Karslı, Y., Chavarría-K, C., 2019, Journal of Astrophysics and Astronomy, 40,33

Akkaya Oralhan, Í., Michel, R., Karslı, Y., Cakmak, H., Sung, H., Karataş, Y, 2020, Astronomische Nachritten, 341,44

Alonso-Santiago, J., Negueruela, I., Marco, A., Tabernero, H.M., Gonzalez-Fernandez,C., Castro, N., 2017, MNRAS, 469, 1330

Alonso-Santiago, J., Negueruela, I., Marco, A., Tabernero, H.M., and Castro, N., 2020, arXiv:2009.124118v, Astronomy and Astrophysics, ?,? (A20)

Anderson, R.I., Eyer, L., Mowlavi, N., 2013, MNRAS, 434, 2238

Arellano Ferro, A., Giridhar, S., Rojo, Arellano, E., 2003, RevMexAA, 39, 3

Bastian N., de Mink S. E., 2009, MNRAS, 398, L11

Benedict, G.F. et al., 2007, Astronomical Journal, 133, 1810

Bertelli, G., Bressan, A., Chiosi, C., Fagotto, F., \& Nasi, E., 1994, A\&AS , 106, 275

Bland-Hawthorn, J. and Ortwin Gerhard, O. 2016, Annual Review of Astron and Astrophys, 54, 529

Bonatto Ch., Bica E., Girardi L., 2004, Astronomy and Astrophysics, 415, 571

Bonatto, Ch., Bica, E., 2007, Astronomy and Astrophysics, 473, 445

Bovy, J., 2015, ApJS, 216, 29

Bressan A., Marigo, P., Girardi L., Salasnich, B., Dal Cero, C., Rubele, S., Nanni, A., 2012, MNRAS, 427, 127

Brown, A. G. A., Vallenari, A., Prusti, T. et al., 2018, Astronomy and Astrophysics, 616, 1G

Brown, A. G. A., Vallenari, A., Prusti, T. et al., 2020, 2020arXiv201201533G 
Brunthaler, A., Reid, M.J., Menten, K.M., et.al. 2011, AN, 332, No.5, 461

Bukowiecki, L., Maciejewski, G., Konorski, P., Strobel, A., 2011, AcA 61, 231

Cantat-Gaudin, T., Jordi, C., Vallenari, A., Bragaglia, A., Balaguer-Nunez, L., Soubiran, C., Bossini, D., Moitinho, A., Castro-Ginard, A., Krone-Martins, A., and 3 coauthors., 2018, Astronomy and Astrophysics, 618, 93

Cantat-Gaudin, T., Anders, F., Castro-Ginard, A., Jordi, C., Romero-Gómez, M., Soubiran, C., Casamiquela, L., Tarricq, Y., Moitinho, A., Vallenari, A., Bragaglia, A., KroneMartins, A., Kounkel, M., 2020, Astronomy and Astrophysics, 640, 1

Carney, B. 2001, Star Clusters, Saas-Fee Advanced Course 28, Lecture Notes 1998, Swiss Society for Astrophysics and Astronomy, eds. L. Labhardt and B. Binggeli (Berlin: Springer-Verlag) pp. 1-222

Carraro, G., Costa, E., Ahumada, J.A., 2010, Astronomical Journal, 140, 954

Chen, X. F. and Han, Z. W., 2009, MNRAS, 395, 1822

Chen, X., de Grijs, R., Deng, L., 2010, MNRAS, 464, 119

Chiosi, C., Bertelli, G., Bressan, A., 1992, Annual Review of Astron and Astrophys, 30, 235

Dutra, C.M., Santiago, B.X., Bica, E., 2002, Astronomy and Astrophysics, 383, 219

Ekström, S., Georgy, C., Eggenberger, P., Meynet, G., Mowlavi, N., Wyttenbach, A., Granada, A., Decressin, T., Hirschi, R., Frischknecht, U., Charbonnel, C., Maeder, A., Astronomy and Astrophysics, 2012, 537,146 (E12)

Fernie, J.D., 1963, Astronomical Journal, 68, 780

Ferraro, F.R., 2016, Star Clusters and Black Holes in Galaxies across Cosmic Time, Proceedings of the International Astronomical Union, IAU Symposium, Volume 312, pp. 171-180.

Fiorentino, G., Lanzoni, B., Dalessandro, E., et al.,2014, Astrophysical Journal, 783, 34

Fitzgerald, M.P., 1970, Astronomy and Astrophysics, 4, 234

Gilliland, R. L., Bono,G., Edmonds, P. D., et al.,1998, Astrophysical Journal, 507, 818

Girardi, L., Bertelli, G., Bressan, A., Chiosi, C., Groenewegen, M.A.T., Marigo, P., Salasnich, B., Weiss, A., 2002, Astronomy and Astrophysics, 391, 195

Georgy, C.,Ekström, S., Granada, A., Meynet, G., Mowlavi, N., Eggenberger, P., Maeder, 2013, Astronomy and Astrophysics, 553, 24 (G13)

Guetter, H. H., \& Vrba, F. J. 1989, Astronomical Journal, 98, 611

Hills, J. and Day, C.,1976, Astron. Lett., 17, 87

Johnson, D. R. H., \& Soderblom, D. R., 1987, Astronomical Journal, 93, 864
Kepley, A., Morrison, H.L., Helmi, A., Kinman, T.D., et al., 2007, Astronomical Journal, 134, 1579

Kharchenko, N.V., Piskunov, A.E., Schilbach, E., Röser, S., Scholz, R.D., 2013, Astronomy and Astrophysics, 558, 53

Kilkenny, D., Van Wyk, F., Roberts, G., Marang, F., Cooper, D., 1998, MNRAS, 294, 93

King, I., 1966, Astronomical Journal, 71, 64

Koornneef, J., 1983, Astronomy and Astrophysics, 128, 84

Lazovik, Y.A., Rastorguev, A.S., 2020, Astronomical Journal, 160, 2020

Li, C., de Grijs R., Deng L., Milone A. P., 2017, Astrophysical Journal, 844, 119

Lim, B., Sung, H., Bessell, M. S., Karimov, R.,Ibrahimov, M. 2009, JKAS, 41, 161

Lim, B., Sung, H., Kim, J.S., Bessell, M., Park, B-G., 2014, MNRAS, 443, 454

Lim, B., Sung, H., Kim, J.S., Bessell, M.S., Hwang, N., Park, B-G. 2016, Astrophysical Journal, 831, 116

Lim, B., Rauw, G., Nazé, Y., Sung, H., Hwang, N., Park, B-G., 2019, NatAs, 3, 76

Lindegren, L., Hernandez, J., Bombrun, A., Klioner, S. et al., 2018, Astronomy and Astrophysics, 616, A2

Lindegren, L., Hernandez, J., Bombrun, A., Klioner, S. et al., 2020, 2020arXiv201201533G

Lohr, M. E., Negueruela, I., Tabernero, H. M., Clark, J. S., Lewis, F. and Roche, P. 2018, MNRAS, 478, 3825 (L18)

Lombardi, Jr., J. C., Warren, J. S., Rasio, F. A., Sills, A., and Warren, A. R., 2002, Astrophysical Journal, 568, 939

Maciejewski, G., \& Niedzielski, A. 2007,Astronomy and Astrophysics, 467, 1065

McCrea, W. H., 1964, MNRAS, 128, 147

Marco, A., Negueruela, I., Gonzalez-Fernandez, C., Maiz Apellaniz, J., Dorda, R., Clark, J.S., 2014, Astronomy and Astrophysics, 567, 73

Marigo, P., Girardi, L., Bressan, A., et al., 2008, Astronomy and Astrophysics, 482, 883

Mermilliod, J.-C., 1981, Astronomy and Astrophysics, 97, 235

Menzies, J. W., Marang, F., Laing, J. D., Coulson, I. M., Engelbrecht, C. A., 1991, MNRAS, 248, 642

Molina Lera, J.A, Baume, G., Gamen, R., 2018, MNRAS, 480, 2386

Negueruela, I., \& Marco, A. 2012, Astronomical Journal, 143, 46 (N12)

Negueruela, I., Clark, J. S., Dorda, R., González-Fernández, C., Marco, A., Monguio, M., 2016, Vol.507, page 75 , Edited by Ian Skillen, Marc Balcells, and Scott Trager. ASP Conference Series, San Francisco: Astronomical Society of the Pacific (ASPC). 
Negueruela, I., Monguio, M., Marco, A., Tabernero, H.M., Gonzalez-Fernandez, C., Dorda, R., 2018, MNRAS, 477,2976

Sampedro, L., Dias, W.S., Alfaro, E.J., Monteiro, H., Molina, A., 2017, MNRAS, 470, 3937

Sandage, A.R., 1953, Astronomical Journal, 58, 61

Schmidt-Kaler, Th. 1982, in Landolt-Bornstein, Numerical Data and Functional Relationships in Science and Technology, New Series, Group VI, Vol.2b, eds. K. Schaifers \& H. H. Voigt (Berlin: Springer), p. 14 (SK82)

Schönrich R., Binney J., Dehnen, W., 2010, MNRAS, 403, 1829

Sung, H., Bessell, M.S., See-Woo, L., 1997, Astronomical Journal, 114, 2644

Sung, H., \& Bessell, M. S. 1999, MNRAS, 306, 361

Sung, H., Lim, B., Bessell, M.S. Kim, J.S., Hur, H., Chun, M-Y., Park, B-G., 2013, JKAS, 46, 97 (S13)

Tadross, A. L. 2008, MNRAS, 389, 285

Tout, C. A., Aarseth, S. J., Pols, O. R., Eggleton, P. P., 1997, MNRAS, 291, 732 